\title{
Postappendectomy intra-abdominal abscess: a therapeutic approach
}

\author{
Arkadi Gorenstein, Gaby Gewurtz, Francis Serour, Eli Somekh
}

\begin{abstract}
Four hundred and sixty two children were operated on between January 1989 and June 1993 for acute appendicitis; 10 developed an intra-abdominal abscess. Intra-abdominal abscesses were accompanied by fever above $38^{\circ} \mathrm{C}$ (nine out of 10 patients) and leucocytosis (mean leucocyte count $\left.20.3 \times 10^{9} / 1\right)$ and were detected sonographically four to 14 days after operation. Management included intravenous administration of antibiotics effective against both aerobes and anaerobes, and follow up with serial sonographic studies. Eight patients responded favourably to antibiotic treatment without any drainage procedure, with gradual shrinkage and collapse of abscesses. Two patients, in whom enlargement of collection was demonstrated on serial sonographic examination, eventually underwent percutaneous drainage under sonographic control. These results suggest that some paediatric patients with an intraabdominal abscess after appendicectomy and who are followed up closely by sonography may be managed successfully with appropriate antibiotics alone.

(Arch Dis Child 1994; 70: 400-402)
\end{abstract}

Intra-abdominal abscess is one of the major complications of acute appendicitis in children that has caused substantial morbidity and mortality in the past. ${ }^{1}$ Improvements in the outcome of intra-abdominal abscess have been due to current management with antibiotics directed against both aerobes and anaerobes, early and precise diagnosis by ultrasound and/or computed tomography, and percutaneous drainage under ultrasound and computed tomography control. While appropriate antibiotic treatment has been important in the treatment of intra-abdominal abscess, drainage of the abscess has served as the critical step in management.

We present our experience in managing intra-abdominal abscess after appendicectomy and suggest criteria for conservative treatment alone compared with percutaneous drainage procedure.

\section{Patients and methods}

Four hundred and sixty two children aged 2.5 to 16 years were operated on for presumed acute appendicitis at the Edith Wolfson Medical Center between January 1989 and June 1993. All received intravenous prophylactic treatment with gentamicin and metronidazole. Patients with clinically suspected peritonitis preoperatively received intravenous ampicillin, gentamicin, and metronidazole. Antibiotics were continued for five to seven days if perforation was evident or suspected at operation. Gentamicin plasma concentrations were monitored and maintenance doses were adjusted to provide therapeutic plasma concentrations. Patients with prolonged postoperative fever, abdominal pain, or leucocytosis were routinely examined by abdominal ultrasound.

\section{Results}

Intra-abdominal abscesses were diagnosed in 10 children (eight boys and two girls). Mean age was 8 years (range 3-14 years). Seven patients received perioperative triple antibiotics (fig 1). The diagnosis was perforated appendicitis in six cases, phlegmonousgangrenous appendicitis in three cases, and normal appendix in one case. In seven patients intraoperative peritoneal fluid samples were cultured. Four were sterile while isolates grown from the other three patients were all sensitive to the administered antibiotics. The clinical findings are summarised in the table.

Intra-abdominal abscess was diagnosed four to 14 days after operation (mean $7 \cdot 8$ days). In all patients except one the temperature was above $38^{\circ} \mathrm{C}$ at diagnosis. The mean (range) leucocyte count was $20.3(10 \cdot 1-36 \cdot 5) \times 10^{9} / 1$. In four children the fever and leucocytosis persisted from the time of operation until diagnosis. In the other six, fever subsided after operation with a recurrence of temperature and leucocytosis with development of intra-abdominal abscess. Diagnosis of intra-abdominal abscess

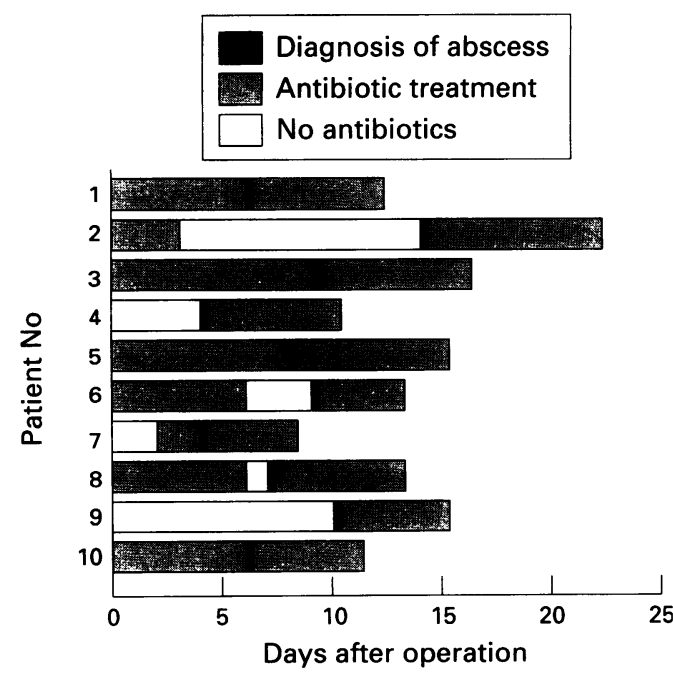

Figure 1 Antibiotic treatment of patients with intraabdominal abscess. 


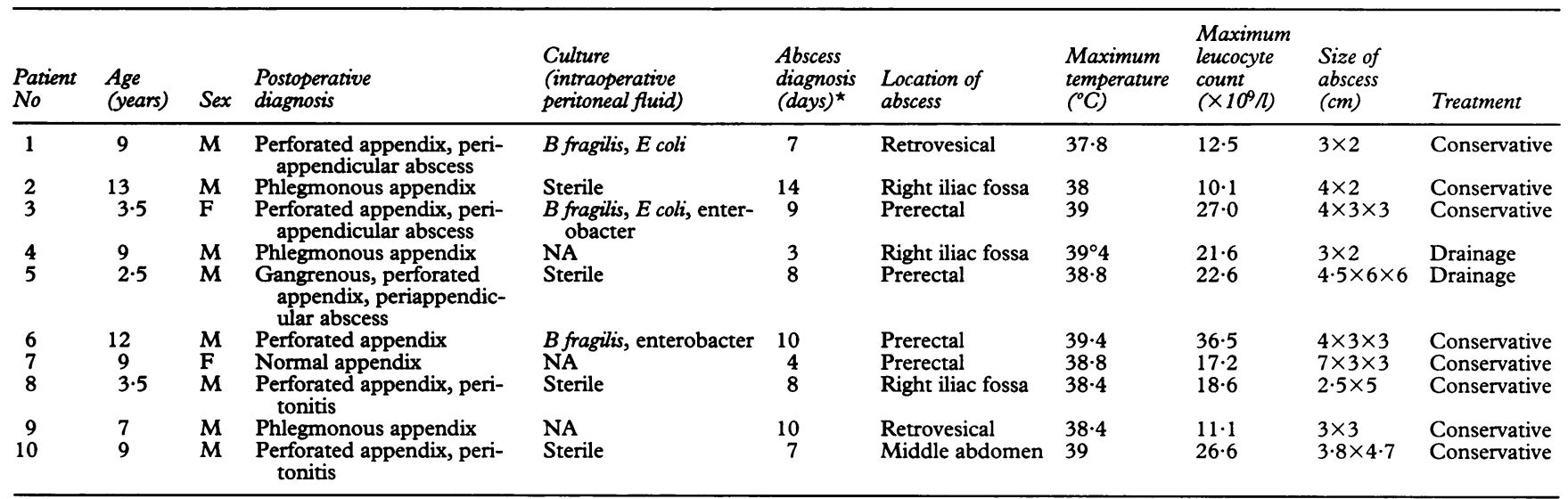

${ }^{\star}$ Days after appendectomy. NA $=$ not available.

was established by ultrasound examination and was localised in the prerectal space (four cases), right iliac fossa (three cases), mid-abdomen (two cases), and retrovesical area (one case).

Basic treatment was intravenous ampicillin, gentamicin, and metronidazole. In five patients this was a continuation of perioperative treatment, four having been initially treated with five days of antibiotics postoperatively and the same antibiotics were restarted after intra-abdominal abscess was demonstrated. In two patients antibiotic treatment was begun after intraabdominal abscess was diagnosed (fig 1). Repeated ultrasound examinations measuring collection size in three dimensions were obtained after intra-abdominal abscess was diagnosed and served as an important criterion for assessing response to treatment in addition to clinical examination and laboratory investigations. Enlargement of intra-abdominal abscess was an indication for changing antibiotics in one patient and for percutaneous drainage under ultrasound control in two. The pus cultured grew Escherichia coli and Bacteroides fragilis (patient 4 ) and $E$ coli (patient 5). The isolates were sensitive to the antibiotics administered before drainage. All 10 patients recovered, eight with antibiotic treatment only and two after percutaneous drainage. None required a second laparotomy or an open drainage procedure. Clinical follow up for at least six months after discharge did not reveal any sign of relapse or reformation of the abscess.

\section{Discussion}

Intra-abdominal abscess is still an important complication of acute appendicitis in children whose rate varies from $1 \cdot 8 \%$ to $6 \%$ after perforated appendicitis ${ }^{2} 3$ and $0 \cdot 8 \%$ after all forms of acute appendicitis. ${ }^{4}$ In spite of early broad spectrum antibiotics, drainage of the abscess has remained the main route of management. ${ }^{5}$ Recently, percutaneous drainage, a less invasive mode of drainage procedure, has been successfully used and is becoming the preferred method of treatment. ${ }^{6}$ However, published experience using antibiotics alone is very scanty. ${ }^{7}$

In our series, the rate of intra-abdominal abscess after appendicectomy was $2 \cdot 2 \%$. Eight out of the 10 patients were successfully managed by intravenous antibiotics alone, while two underwent percutaneous drainage under sonographic control. Serial ultrasonographic measurement of intra-abdominal abscess served as a reliable guide for assessing response to treatment. In the eight patients who responded well to antibiotics, gradual
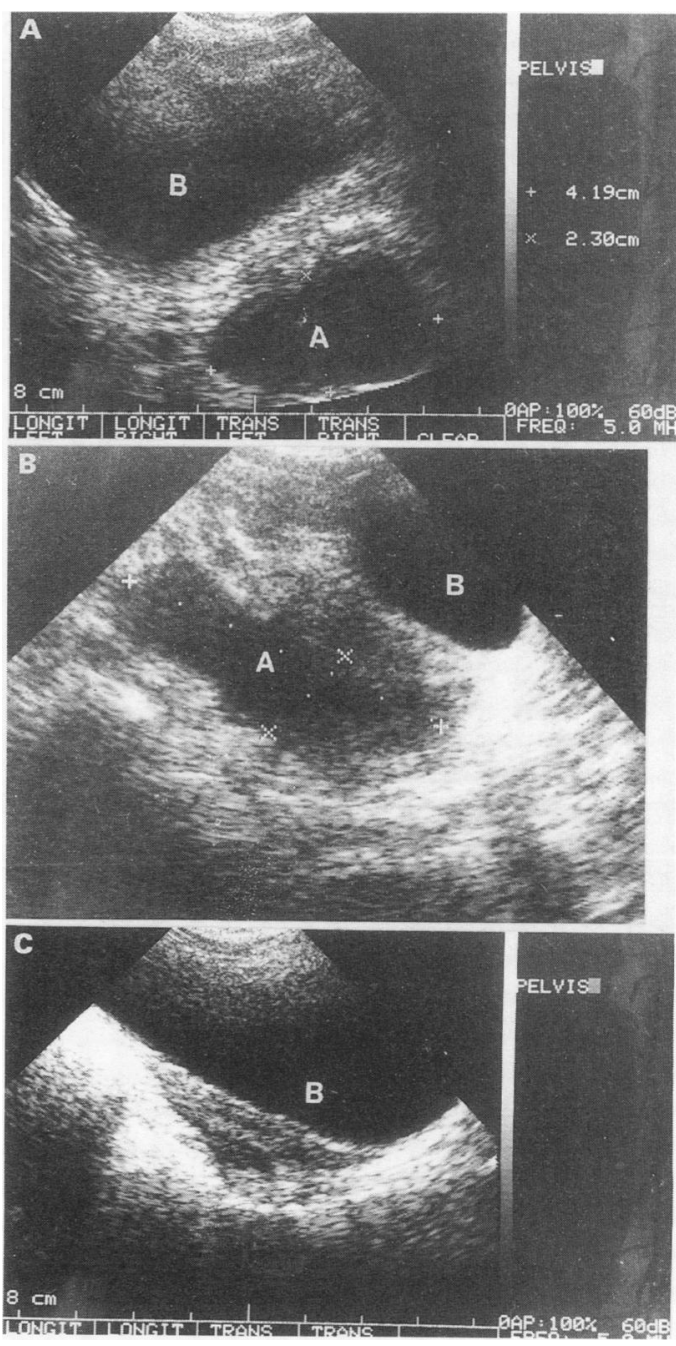

Figure 2 Sonographic follow up of patient 3. (A) Day 9 after appendectomy: longitudinal view through right pelvis showing a large abscess (size $4 \times 3 \mathrm{~cm}$ ). (B) Day 11 after appendectomy: signs of abscess collapse (borders are less sharp and less 'tense'). (C) Day 22 after appendectomy: abscess resolved almost completely with conservative treatment only. ( $A=$ abscess; $B=$ bladder. $)$ 

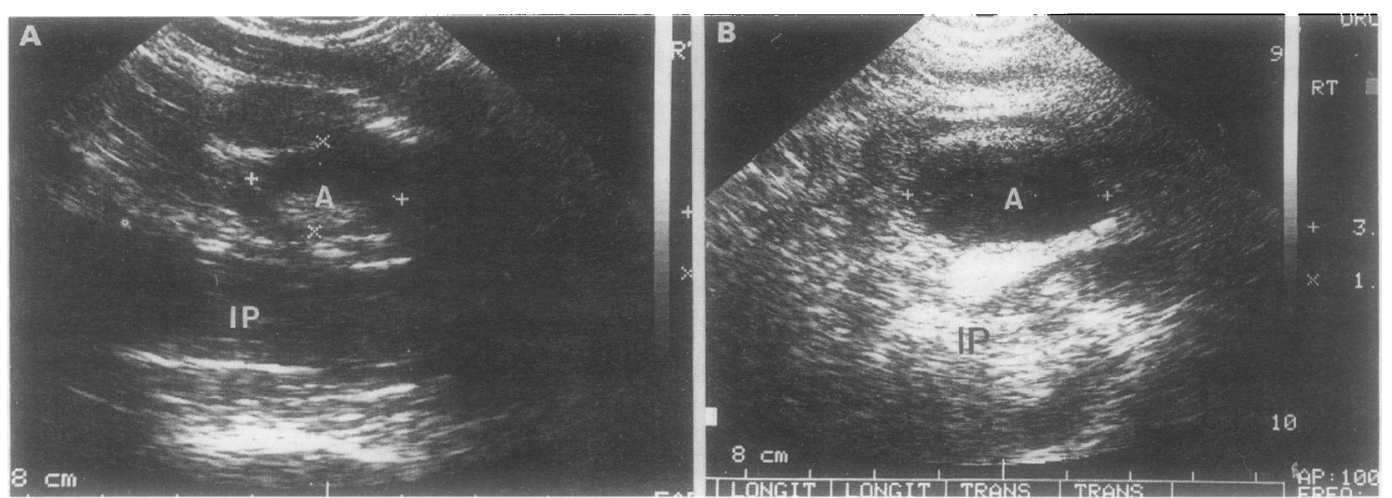

Figure 3 Sonographic follow up of patient 4. (A) Day 4 after appendectomy: longitudinal view through lower abdomen showing non homogenous mass in front of the iliopsoas muscle (size $2 \times 1.3 \mathrm{~cm}$ ). (B) Day 11 after appendectomy: significant abscess enlargement of abscess with evidence of increased tension (the abscess was percutaneously drained one day later $)$. ( $A=$ abscess; $I P=$ iliopsoas muscle. $)$

shrinkage and collapse of the abscess was evident (fig 2). This was accompanied by improvement in the patient's general condition despite persistence of fever and leucocytosis. In the two patients who did not show clinical improvement, ultrasound evidence of progressive enlargement of the intra-abdominal abscess eventually necessitated percutaneous drainage (fig 3). On the other hand, traditional criteria such as fever and leucocyte count did not always serve as good indicators of response to antibiotics. They persisted in two out of eight patients in spite of shrinkage of the intraabdominal abscess. Other clinical criteria namely age, sex, abscess size and location, and time interval from surgery to diagnosis were not helpful in identifying the patients who did not respond to conservative treatment.

The diagnosis of an intra-abdominal abscess was made by ultrasound and/or computed tomography. These techniques are very helpful for differentiating abscesses from fluid collections. However, the diagnosis could be confirmed only after obtaining samples by percutaneous drainage or by laparotomy. However, we consider a more practical and less invasive approach could be close follow up, both clinical and sonographic.

These results suggest that some paediatric patients with an intra-abdominal abscess after appendicectomy may be successfully managed conservatively with appropriate antibiotics alone. Alterations in ultrasound appearance of the intra-abdominal abscess together with clinical data may be a good predictor of outcome. Enlargement of a collection in spite of antibiotic treatment is an indication for the percutaneous drainage procedure. However, this approach should be reserved for patients who are not critically ill, are followed up closely and carefully, and in whom serial sonographic studies can be obtained. By no means should sonographic follow up replace clinical judgment and the general state of the patient should still be the final arbiter of whether a treatment modality is progressing satisfactorily.

1 Shandling B, Ein SH, Simpson JS, et al. Perforating appendicitis and antibiotics. $\mathcal{F}$ Pediatr Surg 1974; 9: 78-83 2 Samelson SL, Reyes HM. Management of perforated appendicitis in children - revisited. Arch Surg 1987; 122 691-6.

3 Burnweit C, Bilik R, Shandling B, Putnam TC. Primary closure of contaminated wounds in perforated appendicitis. F Pediatr Surg 1991; 26: 1362-5.

4 Putnam TC, Gagliano N, Emmens RW. Appendicitis in children. Surg Gynecol Obstet 1990; 170: 527-32.

5 Levison ME, Bush LM. Peritonitis and other intraabdominal infections. In: Mandell GL, ed. Principle and practice of infectious diseases. New York: Churchill-Livingstone, 1990 636-69.

6 Stanley P, Atkinson JB, Reid BS, Gilsanz V. Percutaneous drainage of abdominal fluid collections in children. $A \mathcal{F} R$ 1984; 142: 813-6.

7 Heloury Y, Wetzel O, Bourgoin S, Baron M, Lejus C, Plattner V. Intra-abdominal abscesses localized after appendectomy: is medical or surgical treatment preferable? (British Association of Paediatric Surgeons.) Abstracts of $X X X I X$ Annual International Congress. Leeds, England, 1992: 15 . 